\title{
HERRAMIENTAS 2.0 AL SERVICIO DEL E-LEARNING COLABORATIVO
}

\author{
(SUPPORTING COLLABORATIVE E-LEARNING BASE ON SOFTWARE 2.0)
}

\author{
Susana Trabaldo \\ Nancy Piriz \\ Graciela Lorenzatti \\ Net-learning. Universidad Nacional de San Martín de Argentina. Universidad \\ Tecnológica Nacional de Bs. As. (Argentina)
}

\section{RESUMEN}

Esta experiencia parte del intercambio colaborativo en nuestros cursos y Diplomados de formación en e-learning a los que asisten numerosos miembros de Universidades de Latinoamérica y el Caribe. Allí notamos la gran variedad de programas existentes de educación virtual o en continuo surgimiento y una progresiva aceptación de la modalidad en toda la región.

En esta línea de trabajo surge la idea de crear una comunidad virtual con los miembros de las universidades que ya poseen o desean implementar proyectos de e-learning, con el fin de intercambiar conocimientos y elaborar un producto colaborativo.

La autoría del proyecto pertenece a Susana Trabaldo y Nancy Piriz. La coordinación general estuvo a cargo de Graciela Lorenzatti, y en su desarrollo colaboraron Beatriz Aquino y Edgar Salgado García.

Palabras clave: herramientas 2.o, comunidad virtual, wiki, aprendizaje colaborativo.

\begin{abstract}
This experience arises from courses on e-learning implementation aimed at members of corporations and Universities in Latin America and the Caribbean. The interest in these courses confirms the existence of a variety of virtual education programs in the region and, consequently, the growing acceptance of e-learning.

Universities have been developing new experiences and research projects that meet the population's new educational needs, and working on new teaching and learning programs, that allow their countries to become more competitive.
\end{abstract}


In this context, it was decided to create a virtual community of members belonging to universities that have been implementing or wish to start e-learning projects, with the purpose of constructing knowledge and elaborating a collaborative product.

The project was directed by Susana Trabaldo and Nancy Piriz. The general coordination was in charge of Graciela Lorenzatti, and Beatriz Aquino and Edgar Salgado García were active collaborators.

Keywords: Web 2.o, virtual community, wiki, collaborative learning.

\section{FUNDAMENTACIÓN: GRUPOS Y VINCULOS EN COMUNIDADES VIRTUALES}

\section{Grupo, un universo cotidiano: lo singular y lo colectivo}

Hablar acerca de grupos es ponerle palabras a la experiencia cotidiana, ya que todas las personas participan en diferentes grupos a lo largo de la vida.

GRUPO es un estar-con-otros y si ese estar se despliega dentro de un campus virtual tanto la tarea como el grupo se encuadran en un contexto que reúne características particulares.

La etimología del término permite alguna aproximación a estas particularidades. Originalmente proviene de la voz "Kruppa", que en alemán significa masa redondeada, forma circular. De allí deriva "grop", del provenzal "nudo" y se llega al italiano "groppo" empleado para nombrar los conjuntos pictóricos renacentistas. De esto se desprenden dos conceptos:

- círculo, forma que generalmente adoptan las reuniones grupales;

- nudo, que conlleva la idea de cohesión.

La forma circular hace pensar en una posición equidistante de todos los miembros y a su vez en la creación de un espacio contorneado por ellos. Algo se anuda y se desanuda en ese espacio.

Estos dos conceptos refieren al grupo como un conjunto humano con un sistema de relaciones particulares donde operan determinantes sociales, cosmovisiones individuales que necesariamente confrontan, cargas afectivas que se depositan y circulan entre los integrantes, formas de comunicación que facilitan u obstaculizan 
la formación de vínculos y la existencia de un proceso mediante el cual un conjunto inicial de individuos deviene grupo.

Todo grupo tiene organizadores internos: la tarea y la mutua representación de los integrantes; se define como una estructura de acción y de operación, y actúa en función de las necesidades y su satisfacción.

Considerado desde esta óptica, el grupo permite la formación de vínculos entre los individuos y el despliegue de lo singular y lo colectivo mediante procesos de comunicación y aprendizaje, en un interjuego dialéctico en el que se mantiene la tensión de ambas instancias sin reducir ni la una ni la otra.

Es a través de la comunicación que logra constituirse un vínculo. Y éste es la estructura que sostiene la comunicación. O sea, es una causalidad circular la que permite la comunicación, pero es la comunicación la que constituye el vínculo.

Esto que puede expresarse en pocas palabras implica un proceso complejo de construcción. Mientras se trabaja en una tarea concreta se conforma la red vincular; esto significa un proceso permanente de negociación de significados, porque el grupo está atravesado también por los organizadores culturales.

Cuando se comienza a formar un grupo, cuando se conoce a una persona, es habitual caer en el error de pensar y actuar como si se compartieran los mismos valores e idénticas premisas para conducirse en la vida.

Un grupo es una multiplicidad heterogénea en la que confrontan tantas prácticas como individuos lo integren. Este ha sido el verdadero desafío de la comunidad de práctica: cómo afrontar una tarea colaborativa o una producción conjunta cuando los hacedores provienen de una gran diversidad cultural, profesional, de diferentes localidades, en síntesis, donde no todos comparten los mismos universos de significación.

La heterogeneidad de las historias y de los conocimientos permite tener diferentes resonancias, distintos estilos. Los conceptos que son abstractos se hacen plenos de contenidos cuando se trabaja desde la experiencia que incluye las diferencias y las singularidades. Esto requiere esfuerzo, compromiso y permanentes traducciones de los mensajes que se reciben. Cuando se ponen en juego estas disposiciones positivas entonces la experiencia de grupo, especialmente de grupo colaborativo/cooperativo, permite la puesta en marcha de la creatividad individual, sostenida y potenciada 
por los pares. Por eso la importancia de una buena escucha, de pensar al otro como activo con su condición de saber. Todo esto llevó (en este proyecto) a mantener presente la visualización del mismo, y que fue lo convocante para que se formara la comunidad.

En este grupo con característica cooperativas (Johnson y Johnson, 1999), los integrantes pudieron ayudarse a aprender, discutir, evaluar, atravesar los obstáculos incorporando diferentes miradas, aumentar los logros, mejorar las relaciones personales descubriendo la importancia de completar el conocimiento individual con la práctica y con el conocimiento de los otros, de poder operar con los otros. Es decir, se trabajó en una tarea con dos niveles:

- uno explícito, que incluye un objeto de estudio, problemas que resolver, herramientas de trabajo, pautas y metodología clara y conocida por todos.

- $\quad$ uno implícito, que implica la formación y sostén de la red vincular la cual es soporte del aprendizaje, porque (en sentido amplio) todo vínculo incluye procesos de comunicación y aprendizaje que dan lugar a una desestructuración de modelos incorporados permitiendo resignificar la realidad y reaprender, adaptándose activamente al contexto.

Esta dinámica dentro de un grupo supone un interjuego de roles complementarios, coexistentes y alternativos que hacen a la eficacia de la tarea en cada aquí y ahora. Una mayor heterogeneidad de los integrantes supone una mejor producción porque se logrará construir desde una multiplicidad enriquecedora.

El diagrama siguiente muestra las flechas en un movimiento de ida y vuelta en direcciones opuestas y complementarias que representan la dinámica grupal con fines a la tarea prescripta, tarea como objetivo y como acción, en su doble faz de construir el wikilibro mientras se construía el grupo.

\section{COMUNICACIÓN Y ENTENDIMIENTO}

Comúnmente cuando se habla de comunicación se piensa en el esquema básico de emisor, receptor, canal, código común y mensaje, todo incluido en un contexto. Estos son los elementos elementales sin los cuales no existiría la posibilidad de comunicarse. 
¿Qué se dice cuando se habla con otro? ¿Por qué se puede lograr la comunicación? ¿Desde qué lugar se forma el mensaje? ¿Qué cosas lo constituyen?

Para hablar del mensaje es necesario hacer referencia al lenguaje en tanto conducta social cuya función primaria es la comunicación, por lo tanto elemento de la vida cotidiana. Pensar y hablar se conjugan en la comunicación. Todo pensamiento es comunicable a través del lenguaje. Pero la lengua es una construcción histórica y cultural y aunque como código sea compartida, no siempre se la comparte a nivel de significaciones.

Existe una significación lingüística que otorga capacidad de comunicar significados. La vida cotidiana es el referente de esa significación porque ésta es una creación social. La comunicación entre los individuos y la aprehensión subjetiva de la realidad se encuadran en un universo simbólico legitimado socialmente.

El acto de comunicar es complejo, incluye el contenido explícito, los objetivos, la acción consciente de aquel que emite un mensaje (emisor) y del que lo recibe (receptor).

Se puede decir que el mensaje se encuentra en medio de dos ejes: a) la estructura de la narración y b) la connotación, el significado (el mensaje manifiesto y el implícito). Igualmente se pueden analizar tres niveles de significación en los que influye el contexto:

- Significación lingüística: lo que se entiende cuando se conoce la lengua (código compartido).

- Significación referencial: respecto a objetos reales.

- Significación completa: que da cuenta de la captación del contenido, de los diversos efectos de sentido.

Si la producción del lenguaje es social, ¿cómo se produce la entrada del sujeto en el significado, que también es social? Ambos son elementos fundantes de la acción comunicativa.

En los seres humanos hay una sensibilidad innata al contexto que surge a partir de una predisposición a los significados culturales, sociales. El sujeto se incluye en una red familiar en la que es protagonista, se inserta en un contexto y en él se produce la captación (tanto prelingüística y lingüística) del significado social que 
le permiten al niño entrar en la cultura. De esto se deduce que la función precede a la forma, la necesidad de construir significados y organizar la experiencia, es lo que lleva al sujeto a comunicarse. Necesidad de insertarse en una cultura. Aprendizaje en la acción. Conocimiento y praxis son inseparables culturalmente.

A partir de estos elementos todo ser comunica; el gesto, la palabra, el silencio forman parte del mensaje codificado que otro decodifica dentro de un contexto determinado.

Como se puede ver, aquel esquema inicial de emisor, receptor y mensaje se ha complejizado. Cada palabra, gesto, silencio tiene un contenido manifiesto específico dentro de un contexto socio-cultural al que se suma un significado histórico individual que también implica lo latente.

El acto cotidiano y común de comunicarse requiere de un análisis para facilitar la comprensión de aquello que se dice y se actúa, muchas veces sin comprender. Esto incluye los ruidos en la comunicación, los que la mayor parte de las veces surgen en el interior de cada sujeto en tanto intenta decodificar desde sí lo que otro codificó. Aquí se juegan la cultura (en tanto contexto) y la forma en que cada uno la ha internalizado. Pertenecer a una cultura implica estar ligado a un conjunto de historia interconectadas aunque esto no suponga consenso y pueda haber distintos niveles de ruptura que dificulten la acción comunicativa.

La explicación anterior se aplica a toda situación comunicativa, incluidos los espacios de comunicación virtual, que van siendo parte de la vida cotidiana, pero que no por eso se los naturaliza, sino que se continúa investigando respecto a cómo se despliegan las situaciones comunicativas en entornos privados del cara a cara.

\section{Modos de establecer la comunicación en los grupos}

Cuando comienzan las relacionarnos en un grupo también se da un proceso de aprendizaje, que los individuos realizan sin darse cuenta. Por ejemplo, se establece un código en común ya que se empieza a conocer qué cosas se pueden hacer y cuáles no, qué cosas se consideran valiosas o no, qué se espera de cada uno.

Se transcriben algunas expresiones vertidas en el inicio de la tarea, que ejemplifican cómo se iba construyendo el código de la comunidad virtual de esta experiencia... 
"Estuve leyendo los aportes e inquietudes planteadas por el grupo hasta ahora. Tenemos que abocarnos entre todos..."

"Y luego entre TODOS votamos por una de la propuestas".

"Coincido contigo que es necesario que xxx vuelque al foro el desarrollo de la propuesta”.

“Quiero armar un grupo”.

"Debo confesar que yo estoy un poco perdida con el objetivo del trabajo".

"Creo que se impone reunirnos con los moderadores y el resto del equipo, para definir que queremos (o podemos) hacer”.

"Por otro lado, si no entiendo mal la propuesta de las coordinadoras, se trata de que cada uno tome la iniciativa y se haga cargo de un pedacito así que hay para todos".

"Podrían precisar si este grupo constituye un grupo de aprendizaje".

"Desde mi punto de vista (espero me disculpen)".

"Entiendo tu punto de vista".

Es habitual observar en los grupos el tipo de comunicación radial que se da cuando todos se dirigen a un solo integrante, modalidad que al repetirse se instala, empobreciendo las posibilidades de interacción grupal. Es de todos a uno, uno el que pregunta y todos le contestan, en general coincide con el desempeño del rol de liderazgo.

Puede detectarse otra forma de comunicación denominada monopolio de la comunicación en la que se observa a un integrante que habla solo, constituyendo una situación de monólogo. A diferencia de la situación anterior, genera incomodidades en algunos integrantes. Este monopolio puede estar atribuido a una persona o un subgrupo. De este modo, este subgrupo se transforma en la parte más activa, responsable de llevar adelante la comunicación y la tarea, mientras que el resto asume un rol de observación.

Otro tipo de comunicación es la comunicación yuxtapuesta, donde todos hablan simultáneamente. Se da cuando hay situaciones que producen un alto monto de ansiedad. Nadie escucha a nadie, todos desean hablar. 
La comunicación lateral, en cambio, permite la interacción de cada integrante del grupo, pero no simultáneamente, por lo tanto, es mucho más eficaz, permite el intercambio de todos los miembros del grupo y no del predominio de unos sobre otros.

Cuando la comunicación es clara permite expresar nuestras ideas y que el otro las entienda. En cambio, cuando es confusa se dan situaciones de incertidumbre e incomprensión, elevándose la ansiedad.

Una comunicación es clara cuando es completa, directa, explícita, congruente, cuando los integrantes de la relación pueden entenderse, pedir y dar explicación... en la que se verifica si el otro escuchó y entendió lo que se quiso decir y viceversa.

Es confusa cuando se manejan frases incompletas, comunicaciones vagas e impersonales, se generaliza, se dan por sobreentendidos valores y significado de las palabras.

Todo lo expresado no puede saberse a priori, estas situaciones van surgiendo a medida que se trabaja, que surgen pensamientos, se expresan ideas y se confronta desde los propios esquemas referenciales.

En la comunidad de referencia, cuando se fue avanzando en el trabajo se convirtió en un espacio confiable, se pudo construir un código común, preguntar y pedir explicaciones sin temor a la respuesta del otro, entonces surgieron otras frases típicas de esta etapa:

“Podrías ser más específico".

"Tu último mail, no entiendo el mensaje”.

"Cómo puedo subsanar el malentendido".

"Y en la medida que nos sintamos cómodas vamos interviniendo".

"Trataremos entre todos".

“¿Se habrá entendido mal?”

"Fui clara".

“¿Necesitan algo más?” 
Comenzó a construirse la mutua representación interna, habiendo un interés especial en ser entendido por los otros, pudiendo compartir la experiencia. Este es momento de mucha fluidez en la comunicación porque se visualiza claramente el proyecto y cada integrante se siente responsable por sí mismo y por el resto.

\section{PROCESOS COGNOSCITIVOS EN PROPUESTAS DE E-LEARNING COLABORATIVAS}

Los procesos cognoscitivos se conciben como las operaciones que lleva a cabo la mente humana entendida como un constructo teórico, e incluyen diversas fases como la senso-percepción, la codificación, la asimilación a las estructuras previas de conocimiento, el almacenamiento y recuperación de información (Anderson, 2001), así como procesos superiores tales como la abstracción, análisis, síntesis y evaluación de la información (Schunk, 1997).

Desde la perspectiva de las neurociencias cognoscitivas, los procesos de pensamiento no consisten solamente en eventos que tienen lugar en un espacio mental abstracto, sino que son funciones realizadas por el cerebro humano, a partir de la acción de complejas redes neuronales.

En los seres humanos, los procesos cognoscitivos involucran los sistemas interrelacionados de pensamiento y lenguaje. Es decir, el trabajo mental se basa en la relación entre elementos semánticos, que se funden para dar lugar a nuevos significados. Además, este proceso conlleva una estructura lingüística y lógica que responde a ciertas reglas determinadas en parte por la dotación biológica, pero predominantemente por el influjo de la cultura. En consecuencia, los procesos cognoscitivos no pueden entenderse en un vacío, puesto que ocurren dentro de un contexto sociocultural que los condiciona y que permite dar un sentido a la construcción de significados.

¿Cómo la psicología cognoscitiva puede ayudar a comprender los procesos interactivos y de aprendizaje colaborativo que tienen lugar en un entorno virtual colaborativo, en este caso específico, en los "wikis"?

Se parte de una concepción del aprendizaje como un cambio relativamente permanente en las estructuras mentales, la conducta o su potencial a partir de la experiencia, en donde los conocimientos previos y la naturaleza de la tarea que enfrenta el sujeto son variables fundamentales que entran en juego. 
La interacción que se lleva a cabo específicamente en los "wikis", herramienta de trabajo colaborativo que se utiliza en la modalidad de aprendizaje virtual o "elearning", favorece este aprendizaje.

\section{LA EXPERIENCIA DE TRABAJO}

\section{Primeros pasos:}

A principios de 2006 se cursaron 300 invitaciones vía correo electrónico a ex alumnos de los mencionados cursos de e-learning, pertenecientes a instituciones de educación superior, del ámbito nacional y de toda la región, del sector público y del privado. Respondieron afirmativamente 150 personas integrantes de las siguientes Universidades:

De Argentina:

- Universidad de Buenos Aires (UBA)

- Universidad Nacional de San Martín

- Universidad Nacional de Luján

- Universidad de Belgrano en Buenos Aires

- Universidad Tecnológica Nacional (UTN)

- Regional Buenos Aires

- Regional Río Grande

- Regional Paraná

- Universidad Nacional de Lomas de Zamora

- Universidad de Morón

- Universidad Católica de Santiago del Estero

- Universidad Nacional de Rosario

- Universidad Nacional de Catamarca

- Universidad Nacional de Tucumán

- Universidad Nacional de Salta 
De otros países:

Perú

- $\quad$ Pontificia Universidad Católica del Perú

- Universidad Los Ángeles de Chimbote

- Universidad Nacional de Piura

Costa Rica

- Universidad Latinoamericana de Ciencia y Tecnología

Venezuela

- Universidad Fermín Toro - Barquisimeto

Uruguay

- Ministerio de Educación de la República Oriental del Uruguay

Chile

- Universidad Tecnológica de Chile

Colombia

- Ambientes Virtuales Educativos S. A. Medellín

México

- Colegio Nacional de Educación Profesional Técnica

\section{OBJETIVOS DE LA COMUNIDAD}

- Desarrollar el tema implementación de proyectos de e-learning.

- Conectar a profesionales de Latinoamérica y el Caribe.

- Generar un ambiente de aprendizaje colaborativo.

- Promover la creación de conocimiento.

- Elaborar un wikilibro. 


\section{¿POR QUÉ UN WIKILIBRO?}

Se pensó en la elaboración de un wikilibro ya que esta es una herramienta de trabajo colaborativo en la web que no requiere de un software especial para editar contenidos; sólo basta el navegador web. Tampoco precisa conocimientos de lenguaje de programación, su uso es sencillo y sus contenidos pueden ser editados por numerosos usuarios de forma independiente, desde cualquier computadora desde cualquier lugar.

Esta herramienta suele ser comparada con una especie de "hoja en blanco" que se puede editar con sencillez y en la que se pueden crear nuevas páginas web.

\section{ETAPAS EN LA CREACIÓN DE UNA COMUNIDAD VIRTUAL DE APRENDIZAJE}

Para desarrollar esta comunidad se siguió el modelo que plantea la Dra. Gilly Salmon (Salmon, 2004) quien describe los pasos para que el tutor y el equipo de apoyo al participante lleguen a alcanzar una interacción exitosa.

El modelo sugiere el avance gradual de la instrucción hacia un enfoque constructivista, transitando de la atención de necesidades a corto plazo, a su atención a largo plazo, y de un aprendizaje puntual e inmediato a otro holístico. Considera 5 etapas de trabajo que se describen a continuación.

\section{Etapa 1: acceso y motivación}

La tutoría interviene dando la bienvenida, acompañamiento, aliento.

El objetivo en esta etapa es exponer a los participantes al entorno/plataforma y asegurarse de que usen la tecnología con éxito y vean los beneficios que aporta al aprendizaje.

El participante necesita de información y soporte técnico en caso de tener que resolver alguna situación problemática. En esta etapa algunos participantes requieren ayuda técnica individual además de la guía general. Los problemas suelen estar relacionados con la configuración del hardware y/o software, el acceso a internet o problemas con la clave. 


\section{Etapa 2: socialización en línea}

Función de la tutoría: facilitar la socialización en el ambiente de aprendizaje.

En esta etapa los participantes se acostumbran a participar en línea. Hay dos motivos por los que los grupos trabajan juntos: el propio interés y el interés común. El primero puede promoverse a través de factores extrínsecos (incentivos), pero el segundo necesita de confianza y respeto mutuo, que deben crecer en el mismo grupo.

Si un participante no llega a relacionarse bien en la comunidad virtual, se "distanciará" también del objeto de aprendizaje. Para que ésto no ocurra, es necesario que el tutor se involucre activamente y encauce las energías hacia la empresa compartida y el propósito de la comunidad de aprendizaje. El tutor crea una experiencia cultural especial que pertenece a este grupo en este momento y que se da a través de la discusión y la negociación.

\section{Etapa 3: manejo de la información}

Tutoría: soporte para el acceso a los materiales y tareas facilitadoras.

El intercambio de información fluye al igual que los mensajes. Algunos participantes pueden sentirse inundados por el caudal de información del que se dispone en un corto tiempo. Los tutores deben ayudarlos a adquirir independencia, confianza en sí mismos y entusiasmo para trabajar en línea.

Es muy importante que para este momento todos tengan un rol y estén participando activamente. El tutor debe continuar con el diseño de interacción para la participación activa y relaciones en línea que propicien el trabajo conjunto.

En general, es "desordenada" la participación en foros o chats. Es importante que los participantes logren adquirir la habilidad de obtener de las interacciones lo que realmente quieren. Es necesario que los participantes comprendan claramente que pueden alcanzar objetivos de trabajo a través de este tipo de interacción.

\section{Etapa 4: construcción del conocimiento}

Tutoría: facilitación del proceso - seguimiento. 


\title{
HERRAMIENTAS 2.0 AL SERVICIO DEL E-LEARNING COLABORATIVO
}

\author{
(SUPPORTING COLLABORATIVE E-LEARNING BASE ON SOFTWARE 2.0)
}

\author{
Susana Trabaldo \\ Nancy Piriz \\ Graciela Lorenzatti \\ Net-learning. Universidad Nacional de San Martín de Argentina. Universidad \\ Tecnológica Nacional de Bs. As. (Argentina)
}

\section{RESUMEN}

Esta experiencia parte del intercambio colaborativo en nuestros cursos y Diplomados de formación en e-learning a los que asisten numerosos miembros de Universidades de Latinoamérica y el Caribe. Allí notamos la gran variedad de programas existentes de educación virtual o en continuo surgimiento y una progresiva aceptación de la modalidad en toda la región.

En esta línea de trabajo surge la idea de crear una comunidad virtual con los miembros de las universidades que ya poseen o desean implementar proyectos de e-learning, con el fin de intercambiar conocimientos y elaborar un producto colaborativo.

La autoría del proyecto pertenece a Susana Trabaldo y Nancy Piriz. La coordinación general estuvo a cargo de Graciela Lorenzatti, y en su desarrollo colaboraron Beatriz Aquino y Edgar Salgado García.

Palabras clave: herramientas 2.o, comunidad virtual, wiki, aprendizaje colaborativo.

\begin{abstract}
This experience arises from courses on e-learning implementation aimed at members of corporations and Universities in Latin America and the Caribbean. The interest in these courses confirms the existence of a variety of virtual education programs in the region and, consequently, the growing acceptance of e-learning.

Universities have been developing new experiences and research projects that meet the population's new educational needs, and working on new teaching and learning programs, that allow their countries to become more competitive.
\end{abstract}


In this context, it was decided to create a virtual community of members belonging to universities that have been implementing or wish to start e-learning projects, with the purpose of constructing knowledge and elaborating a collaborative product.

The project was directed by Susana Trabaldo and Nancy Piriz. The general coordination was in charge of Graciela Lorenzatti, and Beatriz Aquino and Edgar Salgado García were active collaborators.

Keywords: Web 2.o, virtual community, wiki, collaborative learning.

\section{FUNDAMENTACIÓN: GRUPOS Y VINCULOS EN COMUNIDADES VIRTUALES}

\section{Grupo, un universo cotidiano: lo singular y lo colectivo}

Hablar acerca de grupos es ponerle palabras a la experiencia cotidiana, ya que todas las personas participan en diferentes grupos a lo largo de la vida.

GRUPO es un estar-con-otros y si ese estar se despliega dentro de un campus virtual tanto la tarea como el grupo se encuadran en un contexto que reúne características particulares.

La etimología del término permite alguna aproximación a estas particularidades. Originalmente proviene de la voz "Kruppa", que en alemán significa masa redondeada, forma circular. De allí deriva "grop", del provenzal "nudo" y se llega al italiano "groppo" empleado para nombrar los conjuntos pictóricos renacentistas. De esto se desprenden dos conceptos:

- círculo, forma que generalmente adoptan las reuniones grupales;

- nudo, que conlleva la idea de cohesión.

La forma circular hace pensar en una posición equidistante de todos los miembros y a su vez en la creación de un espacio contorneado por ellos. Algo se anuda y se desanuda en ese espacio.

Estos dos conceptos refieren al grupo como un conjunto humano con un sistema de relaciones particulares donde operan determinantes sociales, cosmovisiones individuales que necesariamente confrontan, cargas afectivas que se depositan y circulan entre los integrantes, formas de comunicación que facilitan u obstaculizan 
la formación de vínculos y la existencia de un proceso mediante el cual un conjunto inicial de individuos deviene grupo.

Todo grupo tiene organizadores internos: la tarea y la mutua representación de los integrantes; se define como una estructura de acción y de operación, y actúa en función de las necesidades y su satisfacción.

Considerado desde esta óptica, el grupo permite la formación de vínculos entre los individuos y el despliegue de lo singular y lo colectivo mediante procesos de comunicación y aprendizaje, en un interjuego dialéctico en el que se mantiene la tensión de ambas instancias sin reducir ni la una ni la otra.

Es a través de la comunicación que logra constituirse un vínculo. Y éste es la estructura que sostiene la comunicación. O sea, es una causalidad circular la que permite la comunicación, pero es la comunicación la que constituye el vínculo.

Esto que puede expresarse en pocas palabras implica un proceso complejo de construcción. Mientras se trabaja en una tarea concreta se conforma la red vincular; esto significa un proceso permanente de negociación de significados, porque el grupo está atravesado también por los organizadores culturales.

Cuando se comienza a formar un grupo, cuando se conoce a una persona, es habitual caer en el error de pensar y actuar como si se compartieran los mismos valores e idénticas premisas para conducirse en la vida.

Un grupo es una multiplicidad heterogénea en la que confrontan tantas prácticas como individuos lo integren. Este ha sido el verdadero desafío de la comunidad de práctica: cómo afrontar una tarea colaborativa o una producción conjunta cuando los hacedores provienen de una gran diversidad cultural, profesional, de diferentes localidades, en síntesis, donde no todos comparten los mismos universos de significación.

La heterogeneidad de las historias y de los conocimientos permite tener diferentes resonancias, distintos estilos. Los conceptos que son abstractos se hacen plenos de contenidos cuando se trabaja desde la experiencia que incluye las diferencias y las singularidades. Esto requiere esfuerzo, compromiso y permanentes traducciones de los mensajes que se reciben. Cuando se ponen en juego estas disposiciones positivas entonces la experiencia de grupo, especialmente de grupo colaborativo/cooperativo, permite la puesta en marcha de la creatividad individual, sostenida y potenciada 
por los pares. Por eso la importancia de una buena escucha, de pensar al otro como activo con su condición de saber. Todo esto llevó (en este proyecto) a mantener presente la visualización del mismo, y que fue lo convocante para que se formara la comunidad.

En este grupo con característica cooperativas (Johnson y Johnson, 1999), los integrantes pudieron ayudarse a aprender, discutir, evaluar, atravesar los obstáculos incorporando diferentes miradas, aumentar los logros, mejorar las relaciones personales descubriendo la importancia de completar el conocimiento individual con la práctica y con el conocimiento de los otros, de poder operar con los otros. Es decir, se trabajó en una tarea con dos niveles:

- uno explícito, que incluye un objeto de estudio, problemas que resolver, herramientas de trabajo, pautas y metodología clara y conocida por todos.

- $\quad$ uno implícito, que implica la formación y sostén de la red vincular la cual es soporte del aprendizaje, porque (en sentido amplio) todo vínculo incluye procesos de comunicación y aprendizaje que dan lugar a una desestructuración de modelos incorporados permitiendo resignificar la realidad y reaprender, adaptándose activamente al contexto.

Esta dinámica dentro de un grupo supone un interjuego de roles complementarios, coexistentes y alternativos que hacen a la eficacia de la tarea en cada aquí y ahora. Una mayor heterogeneidad de los integrantes supone una mejor producción porque se logrará construir desde una multiplicidad enriquecedora.

El diagrama siguiente muestra las flechas en un movimiento de ida y vuelta en direcciones opuestas y complementarias que representan la dinámica grupal con fines a la tarea prescripta, tarea como objetivo y como acción, en su doble faz de construir el wikilibro mientras se construía el grupo.

\section{COMUNICACIÓN Y ENTENDIMIENTO}

Comúnmente cuando se habla de comunicación se piensa en el esquema básico de emisor, receptor, canal, código común y mensaje, todo incluido en un contexto. Estos son los elementos elementales sin los cuales no existiría la posibilidad de comunicarse. 
¿Qué se dice cuando se habla con otro? ¿Por qué se puede lograr la comunicación? ¿Desde qué lugar se forma el mensaje? ¿Qué cosas lo constituyen?

Para hablar del mensaje es necesario hacer referencia al lenguaje en tanto conducta social cuya función primaria es la comunicación, por lo tanto elemento de la vida cotidiana. Pensar y hablar se conjugan en la comunicación. Todo pensamiento es comunicable a través del lenguaje. Pero la lengua es una construcción histórica y cultural y aunque como código sea compartida, no siempre se la comparte a nivel de significaciones.

Existe una significación lingüística que otorga capacidad de comunicar significados. La vida cotidiana es el referente de esa significación porque ésta es una creación social. La comunicación entre los individuos y la aprehensión subjetiva de la realidad se encuadran en un universo simbólico legitimado socialmente.

El acto de comunicar es complejo, incluye el contenido explícito, los objetivos, la acción consciente de aquel que emite un mensaje (emisor) y del que lo recibe (receptor).

Se puede decir que el mensaje se encuentra en medio de dos ejes: a) la estructura de la narración y b) la connotación, el significado (el mensaje manifiesto y el implícito). Igualmente se pueden analizar tres niveles de significación en los que influye el contexto:

- Significación lingüística: lo que se entiende cuando se conoce la lengua (código compartido).

- Significación referencial: respecto a objetos reales.

- Significación completa: que da cuenta de la captación del contenido, de los diversos efectos de sentido.

Si la producción del lenguaje es social, ¿cómo se produce la entrada del sujeto en el significado, que también es social? Ambos son elementos fundantes de la acción comunicativa.

En los seres humanos hay una sensibilidad innata al contexto que surge a partir de una predisposición a los significados culturales, sociales. El sujeto se incluye en una red familiar en la que es protagonista, se inserta en un contexto y en él se produce la captación (tanto prelingüística y lingüística) del significado social que 
le permiten al niño entrar en la cultura. De esto se deduce que la función precede a la forma, la necesidad de construir significados y organizar la experiencia, es lo que lleva al sujeto a comunicarse. Necesidad de insertarse en una cultura. Aprendizaje en la acción. Conocimiento y praxis son inseparables culturalmente.

A partir de estos elementos todo ser comunica; el gesto, la palabra, el silencio forman parte del mensaje codificado que otro decodifica dentro de un contexto determinado.

Como se puede ver, aquel esquema inicial de emisor, receptor y mensaje se ha complejizado. Cada palabra, gesto, silencio tiene un contenido manifiesto específico dentro de un contexto socio-cultural al que se suma un significado histórico individual que también implica lo latente.

El acto cotidiano y común de comunicarse requiere de un análisis para facilitar la comprensión de aquello que se dice y se actúa, muchas veces sin comprender. Esto incluye los ruidos en la comunicación, los que la mayor parte de las veces surgen en el interior de cada sujeto en tanto intenta decodificar desde sí lo que otro codificó. Aquí se juegan la cultura (en tanto contexto) y la forma en que cada uno la ha internalizado. Pertenecer a una cultura implica estar ligado a un conjunto de historia interconectadas aunque esto no suponga consenso y pueda haber distintos niveles de ruptura que dificulten la acción comunicativa.

La explicación anterior se aplica a toda situación comunicativa, incluidos los espacios de comunicación virtual, que van siendo parte de la vida cotidiana, pero que no por eso se los naturaliza, sino que se continúa investigando respecto a cómo se despliegan las situaciones comunicativas en entornos privados del cara a cara.

\section{Modos de establecer la comunicación en los grupos}

Cuando comienzan las relacionarnos en un grupo también se da un proceso de aprendizaje, que los individuos realizan sin darse cuenta. Por ejemplo, se establece un código en común ya que se empieza a conocer qué cosas se pueden hacer y cuáles no, qué cosas se consideran valiosas o no, qué se espera de cada uno.

Se transcriben algunas expresiones vertidas en el inicio de la tarea, que ejemplifican cómo se iba construyendo el código de la comunidad virtual de esta experiencia... 
"Estuve leyendo los aportes e inquietudes planteadas por el grupo hasta ahora. Tenemos que abocarnos entre todos..."

"Y luego entre TODOS votamos por una de la propuestas".

"Coincido contigo que es necesario que xxx vuelque al foro el desarrollo de la propuesta”.

“Quiero armar un grupo”.

"Debo confesar que yo estoy un poco perdida con el objetivo del trabajo".

"Creo que se impone reunirnos con los moderadores y el resto del equipo, para definir que queremos (o podemos) hacer”.

"Por otro lado, si no entiendo mal la propuesta de las coordinadoras, se trata de que cada uno tome la iniciativa y se haga cargo de un pedacito así que hay para todos".

"Podrían precisar si este grupo constituye un grupo de aprendizaje".

"Desde mi punto de vista (espero me disculpen)".

"Entiendo tu punto de vista".

Es habitual observar en los grupos el tipo de comunicación radial que se da cuando todos se dirigen a un solo integrante, modalidad que al repetirse se instala, empobreciendo las posibilidades de interacción grupal. Es de todos a uno, uno el que pregunta y todos le contestan, en general coincide con el desempeño del rol de liderazgo.

Puede detectarse otra forma de comunicación denominada monopolio de la comunicación en la que se observa a un integrante que habla solo, constituyendo una situación de monólogo. A diferencia de la situación anterior, genera incomodidades en algunos integrantes. Este monopolio puede estar atribuido a una persona o un subgrupo. De este modo, este subgrupo se transforma en la parte más activa, responsable de llevar adelante la comunicación y la tarea, mientras que el resto asume un rol de observación.

Otro tipo de comunicación es la comunicación yuxtapuesta, donde todos hablan simultáneamente. Se da cuando hay situaciones que producen un alto monto de ansiedad. Nadie escucha a nadie, todos desean hablar. 
La comunicación lateral, en cambio, permite la interacción de cada integrante del grupo, pero no simultáneamente, por lo tanto, es mucho más eficaz, permite el intercambio de todos los miembros del grupo y no del predominio de unos sobre otros.

Cuando la comunicación es clara permite expresar nuestras ideas y que el otro las entienda. En cambio, cuando es confusa se dan situaciones de incertidumbre e incomprensión, elevándose la ansiedad.

Una comunicación es clara cuando es completa, directa, explícita, congruente, cuando los integrantes de la relación pueden entenderse, pedir y dar explicación... en la que se verifica si el otro escuchó y entendió lo que se quiso decir y viceversa.

Es confusa cuando se manejan frases incompletas, comunicaciones vagas e impersonales, se generaliza, se dan por sobreentendidos valores y significado de las palabras.

Todo lo expresado no puede saberse a priori, estas situaciones van surgiendo a medida que se trabaja, que surgen pensamientos, se expresan ideas y se confronta desde los propios esquemas referenciales.

En la comunidad de referencia, cuando se fue avanzando en el trabajo se convirtió en un espacio confiable, se pudo construir un código común, preguntar y pedir explicaciones sin temor a la respuesta del otro, entonces surgieron otras frases típicas de esta etapa:

“Podrías ser más específico".

"Tu último mail, no entiendo el mensaje”.

"Cómo puedo subsanar el malentendido".

"Y en la medida que nos sintamos cómodas vamos interviniendo".

"Trataremos entre todos".

“¿Se habrá entendido mal?”

"Fui clara".

“¿Necesitan algo más?” 
Comenzó a construirse la mutua representación interna, habiendo un interés especial en ser entendido por los otros, pudiendo compartir la experiencia. Este es momento de mucha fluidez en la comunicación porque se visualiza claramente el proyecto y cada integrante se siente responsable por sí mismo y por el resto.

\section{PROCESOS COGNOSCITIVOS EN PROPUESTAS DE E-LEARNING COLABORATIVAS}

Los procesos cognoscitivos se conciben como las operaciones que lleva a cabo la mente humana entendida como un constructo teórico, e incluyen diversas fases como la senso-percepción, la codificación, la asimilación a las estructuras previas de conocimiento, el almacenamiento y recuperación de información (Anderson, 2001), así como procesos superiores tales como la abstracción, análisis, síntesis y evaluación de la información (Schunk, 1997).

Desde la perspectiva de las neurociencias cognoscitivas, los procesos de pensamiento no consisten solamente en eventos que tienen lugar en un espacio mental abstracto, sino que son funciones realizadas por el cerebro humano, a partir de la acción de complejas redes neuronales.

En los seres humanos, los procesos cognoscitivos involucran los sistemas interrelacionados de pensamiento y lenguaje. Es decir, el trabajo mental se basa en la relación entre elementos semánticos, que se funden para dar lugar a nuevos significados. Además, este proceso conlleva una estructura lingüística y lógica que responde a ciertas reglas determinadas en parte por la dotación biológica, pero predominantemente por el influjo de la cultura. En consecuencia, los procesos cognoscitivos no pueden entenderse en un vacío, puesto que ocurren dentro de un contexto sociocultural que los condiciona y que permite dar un sentido a la construcción de significados.

¿Cómo la psicología cognoscitiva puede ayudar a comprender los procesos interactivos y de aprendizaje colaborativo que tienen lugar en un entorno virtual colaborativo, en este caso específico, en los "wikis"?

Se parte de una concepción del aprendizaje como un cambio relativamente permanente en las estructuras mentales, la conducta o su potencial a partir de la experiencia, en donde los conocimientos previos y la naturaleza de la tarea que enfrenta el sujeto son variables fundamentales que entran en juego. 
La interacción que se lleva a cabo específicamente en los "wikis", herramienta de trabajo colaborativo que se utiliza en la modalidad de aprendizaje virtual o "elearning", favorece este aprendizaje.

\section{LA EXPERIENCIA DE TRABAJO}

\section{Primeros pasos:}

A principios de 2006 se cursaron 300 invitaciones vía correo electrónico a ex alumnos de los mencionados cursos de e-learning, pertenecientes a instituciones de educación superior, del ámbito nacional y de toda la región, del sector público y del privado. Respondieron afirmativamente 150 personas integrantes de las siguientes Universidades:

De Argentina:

- Universidad de Buenos Aires (UBA)

- Universidad Nacional de San Martín

- Universidad Nacional de Luján

- Universidad de Belgrano en Buenos Aires

- Universidad Tecnológica Nacional (UTN)

- Regional Buenos Aires

- Regional Río Grande

- Regional Paraná

- Universidad Nacional de Lomas de Zamora

- Universidad de Morón

- Universidad Católica de Santiago del Estero

- Universidad Nacional de Rosario

- Universidad Nacional de Catamarca

- Universidad Nacional de Tucumán

- Universidad Nacional de Salta 
De otros países:

Perú

- $\quad$ Pontificia Universidad Católica del Perú

- Universidad Los Ángeles de Chimbote

- Universidad Nacional de Piura

Costa Rica

- Universidad Latinoamericana de Ciencia y Tecnología

Venezuela

- Universidad Fermín Toro - Barquisimeto

Uruguay

- Ministerio de Educación de la República Oriental del Uruguay

Chile

- Universidad Tecnológica de Chile

Colombia

- Ambientes Virtuales Educativos S. A. Medellín

México

- Colegio Nacional de Educación Profesional Técnica

\section{OBJETIVOS DE LA COMUNIDAD}

- Desarrollar el tema implementación de proyectos de e-learning.

- Conectar a profesionales de Latinoamérica y el Caribe.

- Generar un ambiente de aprendizaje colaborativo.

- Promover la creación de conocimiento.

- Elaborar un wikilibro. 


\section{¿POR QUÉ UN WIKILIBRO?}

Se pensó en la elaboración de un wikilibro ya que esta es una herramienta de trabajo colaborativo en la web que no requiere de un software especial para editar contenidos; sólo basta el navegador web. Tampoco precisa conocimientos de lenguaje de programación, su uso es sencillo y sus contenidos pueden ser editados por numerosos usuarios de forma independiente, desde cualquier computadora desde cualquier lugar.

Esta herramienta suele ser comparada con una especie de "hoja en blanco" que se puede editar con sencillez y en la que se pueden crear nuevas páginas web.

\section{ETAPAS EN LA CREACIÓN DE UNA COMUNIDAD VIRTUAL DE APRENDIZAJE}

Para desarrollar esta comunidad se siguió el modelo que plantea la Dra. Gilly Salmon (Salmon, 2004) quien describe los pasos para que el tutor y el equipo de apoyo al participante lleguen a alcanzar una interacción exitosa.

El modelo sugiere el avance gradual de la instrucción hacia un enfoque constructivista, transitando de la atención de necesidades a corto plazo, a su atención a largo plazo, y de un aprendizaje puntual e inmediato a otro holístico. Considera 5 etapas de trabajo que se describen a continuación.

\section{Etapa 1: acceso y motivación}

La tutoría interviene dando la bienvenida, acompañamiento, aliento.

El objetivo en esta etapa es exponer a los participantes al entorno/plataforma y asegurarse de que usen la tecnología con éxito y vean los beneficios que aporta al aprendizaje.

El participante necesita de información y soporte técnico en caso de tener que resolver alguna situación problemática. En esta etapa algunos participantes requieren ayuda técnica individual además de la guía general. Los problemas suelen estar relacionados con la configuración del hardware y/o software, el acceso a internet o problemas con la clave. 


\section{Etapa 2: socialización en línea}

Función de la tutoría: facilitar la socialización en el ambiente de aprendizaje.

En esta etapa los participantes se acostumbran a participar en línea. Hay dos motivos por los que los grupos trabajan juntos: el propio interés y el interés común. El primero puede promoverse a través de factores extrínsecos (incentivos), pero el segundo necesita de confianza y respeto mutuo, que deben crecer en el mismo grupo.

Si un participante no llega a relacionarse bien en la comunidad virtual, se "distanciará" también del objeto de aprendizaje. Para que ésto no ocurra, es necesario que el tutor se involucre activamente y encauce las energías hacia la empresa compartida y el propósito de la comunidad de aprendizaje. El tutor crea una experiencia cultural especial que pertenece a este grupo en este momento y que se da a través de la discusión y la negociación.

\section{Etapa 3: manejo de la información}

Tutoría: soporte para el acceso a los materiales y tareas facilitadoras.

El intercambio de información fluye al igual que los mensajes. Algunos participantes pueden sentirse inundados por el caudal de información del que se dispone en un corto tiempo. Los tutores deben ayudarlos a adquirir independencia, confianza en sí mismos y entusiasmo para trabajar en línea.

Es muy importante que para este momento todos tengan un rol y estén participando activamente. El tutor debe continuar con el diseño de interacción para la participación activa y relaciones en línea que propicien el trabajo conjunto.

En general, es "desordenada" la participación en foros o chats. Es importante que los participantes logren adquirir la habilidad de obtener de las interacciones lo que realmente quieren. Es necesario que los participantes comprendan claramente que pueden alcanzar objetivos de trabajo a través de este tipo de interacción.

\section{Etapa 4: construcción del conocimiento}

Tutoría: facilitación del proceso - seguimiento. 
Los esfuerzos realizados en las anteriores etapas preparan a los participantes para el éxito. En esta etapa estos comienzan a interactuar entre sí de modo más expuesto y participativo. Formulan y escriben sus ideas o entendimiento de los temas. Se leen entre sí y se responden. Especialmente pueden compartir discrepancias, ampliar sus puntos de vista y apreciar diferentes perspectivas. Se enriquece la comprensión de conceptos y teorías a través del debate y de ejemplos que dan otros participantes. Este es un momento que debe aprovecharse al máximo.

"Los participantes pueden aprender más entre sí que del material del curso o de lo expresado por el tutor. Lo que aprenden, por supuesto, no es información sino proceso: en especial el proceso cognitivo creativo de proponer ideas, exponerlas a la crítica o a su mejora y tener la oportunidad de darle una nueva forma (o abandonarlas) a la luz de la discusión de sus pares. El aprendizaje no es simplemente activo... es interactivo. Los estudiantes disponen de quien pueden obtener una respuesta individual a sus inquietudes o nuevas ideas y de quien pueden percibir una perspectiva alternativa desafiante. A cambio, ellos pueden contribuir del mismo modo con el aprendizaje de sus colegas (y ellos mismos pueden aprender durante este proceso)" (Rowntree, 1992, p.56).

Este "constructivismo comunitario" pone énfasis en la construcción del conocimiento en grupos y se basa en situaciones reales y experiencias. El uso de la tecnología en red permite el acceso a la comunicación y el compartir tal conocimiento, la oportunidad de presentar y publicar puntos de vista individuales y colectivos, la construcción sobre ideas de otros. Este es el objetivo de la cuarta etapa: desarrollar un aprendizaje colaborativo altamente productivo.

\section{Etapa 5: desarrollo de Metacognición}

Los estudiantes se hacen responsables de su propio aprendizaje.

La función de la tutoría en esta etapa es crear actividades que promuevan el pensamiento crítico, proveer vínculos fuera del aula virtual para que el alumno pueda profundizar y desarrollar su conocimiento sobre el tema de aprendizaje y sobre el modo en que la interacción en línea puede contribuir en su aprendizaje.

Hay razones muy importantes para sostener el aprendizaje en línea, no sólo para la construcción del conocimiento gradual sino también para promover habilidades cognitivas individuales y de reflexión. La metacognición promueve la integración y aplicación de experiencias de aprendizaje. 
Los esfuerzos realizados en las anteriores etapas preparan a los participantes para el éxito. En esta etapa estos comienzan a interactuar entre sí de modo más expuesto y participativo. Formulan y escriben sus ideas o entendimiento de los temas. Se leen entre sí y se responden. Especialmente pueden compartir discrepancias, ampliar sus puntos de vista y apreciar diferentes perspectivas. Se enriquece la comprensión de conceptos y teorías a través del debate y de ejemplos que dan otros participantes. Este es un momento que debe aprovecharse al máximo.

"Los participantes pueden aprender más entre sí que del material del curso o de lo expresado por el tutor. Lo que aprenden, por supuesto, no es información sino proceso: en especial el proceso cognitivo creativo de proponer ideas, exponerlas a la crítica o a su mejora y tener la oportunidad de darle una nueva forma (o abandonarlas) a la luz de la discusión de sus pares. El aprendizaje no es simplemente activo... es interactivo. Los estudiantes disponen de quien pueden obtener una respuesta individual a sus inquietudes o nuevas ideas y de quien pueden percibir una perspectiva alternativa desafiante. A cambio, ellos pueden contribuir del mismo modo con el aprendizaje de sus colegas (y ellos mismos pueden aprender durante este proceso)" (Rowntree, 1992, p.56).

Este "constructivismo comunitario" pone énfasis en la construcción del conocimiento en grupos y se basa en situaciones reales y experiencias. El uso de la tecnología en red permite el acceso a la comunicación y el compartir tal conocimiento, la oportunidad de presentar y publicar puntos de vista individuales y colectivos, la construcción sobre ideas de otros. Este es el objetivo de la cuarta etapa: desarrollar un aprendizaje colaborativo altamente productivo.

\section{Etapa 5: desarrollo de Metacognición}

Los estudiantes se hacen responsables de su propio aprendizaje.

La función de la tutoría en esta etapa es crear actividades que promuevan el pensamiento crítico, proveer vínculos fuera del aula virtual para que el alumno pueda profundizar y desarrollar su conocimiento sobre el tema de aprendizaje y sobre el modo en que la interacción en línea puede contribuir en su aprendizaje.

Hay razones muy importantes para sostener el aprendizaje en línea, no sólo para la construcción del conocimiento gradual sino también para promover habilidades cognitivas individuales y de reflexión. La metacognición promueve la integración y aplicación de experiencias de aprendizaje. 
En esta etapa los participantes son responsables de su propio aprendizaje. Se requieren habilidades distintas: pensamiento crítico y la habilidad de desafiar presupuestos. Es en este momento cuando los participantes se sueltan y se atreven a escribir cosas que antes no hacían, a expresar más sus reflexiones a través del entorno virtual, a examinar sus propios modos de pensar y su proceso de construcción de conocimiento.

\section{PASOS EN LA ELABORACIÓN DEL WIKILIBRO}

Se especifica a continuación cuáles fueron los pasos dados en la creación y desarrollo de la comunidad virtual de aprendizaje de gestores de proyectos de elearning y el recorrido realizado para alcanzar su objetivo: la publicación de un wikilibro.

\section{Primero: Comienzo de la organización y los primeros contactos}

Después de que las Directoras del proyecto definieran el marco conceptual y el problema o tema disparador, designaron a la Coordinadora y estimularon a proponerse como moderadores y a plantear temas centrales a desarrollar. Se procuró que todos pudieran tener acceso rápido y fácil al campus virtual motivándolos a participar en la naciente comunidad virtual.

\section{Segundo: Presentación. Armado de grupos en torno a los ocho temas}

Una vez confirmada la presencia de todos los moderadores, y para favorecer la socialización en línea, la Coordinadora abrió un foro de presentación de todos los miembros de la comunidad para fortalecer el vínculo entre los participantes. Paralelamente fue organizando grupos de trabajo colaborativo a partir de intereses temáticos comunes manifestados por cada uno de los participantes, los que estarían luego regulados por uno o varios moderadores. Así, quedaron conformados ocho grupos de trabajo de acuerdo con los siguientes temas propuestos:

- Inserción estratégica del proyecto en la Universidad: áreas, organigrama, dirección y coordinación, marketing.

- Costos del proyecto: costos fijos y variables, financiación, ROI.

- Tutores: selección y formación, competencias a desarrollar.

- Alumnos: preparación previa, competencias necesarias y acompañamiento. 
- Enfoque pedagógico: programas, contenidos, metodologías.

- Materiales: decisiones acerca de contenidos y materiales.

- Tecnologías: decisiones acerca del entorno tecnológico del proyecto.

- Calidad: evaluación y certificación.

También, durante esta segunda etapa se fueron estableciendo acuerdos de la comunidad a través de normas y reglas de trabajo que los mismos grupos fueron decidiendo. Se entregó un primer instructivo para los moderadores con sugerencias de cronograma, los que a su vez fueron planteando a la Coordinadora dudas y consultas sobre su rol y tarea. Paralelamente, las directoras fueron trabajando en la exploración y organización de la actividad a concretarse en otra etapa: la edición del wikilibro.

\section{Tercero: Manejo de la información}

Algunos moderadores abrieron foros exclusivos o usaron el chat para comunicarse y organizarse en un espacio propio, donde pudieron intercambiar información, preparar el plan de trabajo y organizar la coordinación de su tarea.

Se entregó un segundo instructivo a los moderadores en respuesta a sus planteos y dudas. La comunicación entre la Coordinadora y los moderadores se fue haciendo cada vez más intensa y fluida con el propósito de transmitir seguridad y confianza en el rol. Las Directoras fueron siguiendo atentas el proceso interviniendo cuando fue necesario.

Los moderadores prepararon un plan de trabajo que fue entregado a la Coordinadora para su para su conocimiento, el cual posteriormente fue presentado a sus respetivos grupos.

\section{Cuarto: Generación de conocimiento}

Los moderadores dieron inicio a la actividad grupal, los participantes comenzaron a organizarse a través de los foros. Cada grupo fue creando foros de discusión en torno a los temas específicos y la interacción se hizo más colaborativa.

En la comunicación grupal se fue buscando establecer entendimientos comunes. A través de la colaboración, se fue accediendo al intercambio de informaciones y experiencias, se negociaron nuevos significados, se crearon y recrearon nuevos 
conocimientos. La actividad grupal fue quedando sintetizada en varios resúmenes que fueron elaborados a partir de la interacción en los foros.

En esta etapa cobró gran importancia la función de los moderadores: un total de dieciocho graduados de nuestros cursos se desempeñaron como moderadores grupales, cuya función consistió en:

- Favorecer y motivar el trabajo participativo y colaborativo a través de intervenciones apropiadas que permitan la pluralidad de ideas.

- $\quad$ Promover el intercambio de ideas y mediar ante ideas encontradas.

- Transmitir con claridad la modalidad de trabajo y tiempo de ejecución a través de un plan de trabajo que permitiera a los moderadores organizarse y pensar juntos.

El Plan comprendía: objetivos, metodología de trabajo a usar, temas que se consideraban pertinentes a tratar, tiempos, actividades que podrían favorecer el aporte de los participantes en relación a sus conocimientos teóricos y a su experiencia práctica, el planteo de problemas y el aporte de soluciones, planteos o preguntas disparadoras que pudieran motivar a los integrantes y responsabilidades de quienes participarían en el grupo.

- Plantear actividades que generaran relaciones entre los contenidos y las experiencias de los cursantes.

- Usar los distintos medios de participación y comunicación con los participantes.

- Invitar a la reflexión sintetizando puntos de acuerdo y diferencias. El resumen de las ideas debatidas tenía la importancia de reflejar las opiniones y criterios de cada uno de los miembros del grupo, permitiendo consolidar las reflexiones que resultaban del diálogo a partir de las experiencias.

- Manejar los problemas que fueran surgiendo, con el apoyo y seguimiento de la coordinación, para el aseguramiento de la implementación del proyecto.

\section{Quinto: Integración de la interacción grupal en un producto: el wikilibro}

Para lograr el objetivo común, los participantes se prepararon para elaborar un producto tangible: un wikilibro que contiene la síntesis de los conocimientos alcanzados. 
Cada grupo fue determinando subtemas a desarrollar de acuerdo a las ideas y conocimientos compartidos en los distintos foros de discusión que fueron abriéndose en cada grupo temático.

Se organizó la estructura jerárquica del contenido del wikilibro, iniciándose de este modo la etapa en la que, con la guía de la coordinadora, los grupos fueron volcando sus producciones y avanzando sobre la edición final de los contenidos de su tema.

\section{Revisión general de la producción total}

La coordinadora, con el apoyo de moderadores y participantes voluntarios, supervisó la coherencia y cohesión de toda la producción con el objetivo de mejorar la presentación y organización del trabajo.

A la hora de revisar los capítulos se consideró:

- La colocación de vínculos al pie de la página que enlazaran con el índice del wikilibro, con el tema central del capítulo y con el tema siguiente.

- $\quad$ El sentido y coherencia del contenido.

- La sintaxis de la producción total: elaboración de oraciones, parágrafos, puntuación, empleo de tiempos verbales ( $3^{\circ}$ persona, singular, presente), repeticiones, etc.

- La unificación de títulos y estilo de viñetas.

- La unificación en el lugar y estilo de presentar la bibliografía (orden alfabético, autor, título, editorial, lugar y año; y de links).

- Presentación de cada capítulo: mención de moderadores, una introducción y contenidos. Mención del CV de cada autor de subtemas.

Fue necesario solicitar nuevas contribuciones al desarrollo del wikitexto editado, incluyendo contenidos faltantes, corrigiendo los ya existentes o eliminado con el consentimiento del autor aquello considerado no pertinente a la calidad de la producción.

Con la publicación del wikilibro (www.syncronet.com.ar/mediawiki/) se alcanzaron los objetivos propuestos luego de tres meses de intensa interacción. 


\section{ALGUNAS CONCLUSIONES}

Desarrollar este producto mediante la colaboración en una comunidad virtual fue una rica experiencia de aprendizaje compartido y, a su vez, un gran desafío.

Para lograr el objetivo final fue imprescindible que la tarea estuviera encuadrada en el contexto de un equipo de dinamización (directores, coordinador, moderadores) que garantizara:

- La participación de todos los miembros,

- La generación de intercambios interesantes,

- La discusión de experiencias prácticas,

- $\quad$ El aporte de material teórico que recogiera el estado del arte sobre el tema, y

- La riqueza en la generación de conocimiento.

Como dicho conocimiento es un bien social que se enriquece en la medida en que se comparte con los demás, para coordinar esta comunidad virtual fue necesario ejercitar una meta-mirada que garantizara animar y sostener el proceso colectivo de negociación de significados.

¿Cómo lograr la creación y el mantenimiento de un ambiente de aprendizaje activo y cooperativo? ¿Cómo desarrollar un valioso y productivo intercambio de ideas entre los miembros de la comunidad virtual a partir de sus conocimientos teóricos y por sobre todo desde sus experiencias profesionales? ¿Cómo evitar que el interés de la participación no decayera?

Fue necesario brindar mucho estímulo, acompañamiento y orientación continua a los participantes como también un respetuoso apoyo a la tarea de los moderadores. El fomento a la participación en los foros de discusión, la convocatoria y atracción de quienes no participaban y el monitoreo del cumplimiento de los planes de trabajo grupales también fueron pilares de la tarea de coordinación, la que se vio facilitada por la responsabilidad, el profesionalismo y compromiso con que asumieron su rol los moderadores de cada uno de los ocho grupos.

Si bien hubo algunas bajas entre los moderadores y participantes, ocasionadas por requerimientos laborales personales que restaron tiempo a la participación, o por razones de enfermedad que impidieron continuar en la comunidad virtual, 
no faltó voluntad y decisión de algunos miembros para cubrir esos roles y asumir exitosamente estas tareas, ante la solicitud de la coordinación.

Desde el inicio se evidenciaron fortalezas que favorecieron la coordinación de la comunidad virtual actuando positivamente:

Fortalezas:

- La aceptación voluntaria de los invitados a participar en el proyecto.

- Un manifiesto interés y entusiasmo inicial por participar en la comunidad virtual que hizo que la misma fuera creciendo cada día en número de miembros hasta avanzado su desarrollo.

- La preparación, por parte de los moderadores, de un plan tentativo de trabajo grupal con un banco de temas a los que se sumaron los aportados por los miembros y que sirvió para la orientación de los mismos grupos.

- La elaboración de los primeros resúmenes de las ideas principales debatidas en los foros grupales.

- El diálogo continuo entre moderadores-coordinadora, coordinadora-directora, participantes-coordinadora que fue agilizando el desarrollo de los trabajos grupales.

- El empleo de herramientas colaborativas (foros, chat, correo electrónico, wiki) para facilitar el intercambio, la negociación de significados y respuestas.

- Aciertos, costos personales pero satisfacciones, participaciones y muchas ideas para discutir y aportar.

- Ejecutar decisiones frente a necesidades de cambios de dirección.

Debilidades:

- $\quad$ Apretadas agendas laborales de los participantes que impidieron su continuidad en la comunidad virtual o una participación más activa.

- Altibajos, problemas de salud, cansancio y conflictos.

- Falta de continuidad para mantener el contacto diario en las discusiones.

- Decaimiento del interés por el trabajo en equipo. 
Integrando los principios constructivistas se logró:

- Permitir un procesamiento de información sostenido, no sujeto a la coincidencia de los participantes en el tiempo, que a la vez fue fluido y contribuyó a un análisis más detallado que si la interacción hubiese sido presencial.

- Vincular los conceptos con las experiencias de cada persona, dado que todos fueron participantes de los diplomados y programa de experto en e-learning.

- Aprovechar la motivación intrínseca de los participantes, quienes de forma voluntaria se integraron al trabajo y eligieron los temas de acuerdo con sus intereses personales y profesionales.

- Retroalimentar la tarea de manera constante gracias a la naturaleza del "wiki", el cual lleva un registro de los aportes de cada individuo.

- Elaborar propuestas basadas en la contrastación de experiencias y significados generados por cada uno de los integrantes, de forma que se logró un verdadero "aprendizaje significativo".

Partiendo también del concepto de "zona de desarrollo próximo" (Vigotsky, 1982), según el cual los aprendices deben trabajar en la zona comprendida entre lo que son capaces de hacer actualmente y el potencial que podrían alcanzar mediante la guía de sus compañeros, se evidencia que la experiencia del wikilibro reflejó este proceso, ya que creó un espacio en el que algunos, quizá no tan experimentados, lograron aprender de quienes tenían una mayor trayectoria en los temas de e-learning, cosa que no hubiese sido posible con un proceso de aprendizaje individual.

La actividad no solamente apuntó a un producto, como es el "documento final”, sino también al proceso, es decir, a la experiencia de los usuarios a lo largo de su realización, en donde se encuentra predominantemente la riqueza de esta herramienta. La construcción de un wikilibro es un proceso recursivo, que a manera de "ciclo sin fin" (endless loop) queda abierto al perfeccionamiento continuo.

Si se traslada esta experiencia a un ámbito académico se puede observar que la participación en un wiki va más allá de los roles tradicionales de "maestro" y "aprendiz" ya que contribuye a generar un espacio en el que todos enseñan y aprenden.

Se ha comprobado que las comunidades virtuales constituyen un excelente mecanismo para la transformación de la información y reflexión sobre la práctica en conocimiento en movimiento. 


\section{REFERENCIAS BIBLIOGRÁFICAS}

Abate Daga, M.; Peralta, M. E. (2003). Las teorías del desarrollo psicológico en el ámbito de la educación. Congreso Interoceánico de Estudios Latinoamericanos. Mendoza (Argentina) [en línea] Disponible en: http://ffyl.uncu. edu.ar/ifaa/archivo/IIInteroceanico/ Sujeto/Educativas/Abate.doc [consulta 2006, 2 de febrero].

Kozulin, A. (1998). Los paradigmas vigostkianos y de experiencia de aprendizaje mediado (eam) en la capacitación de docentes. Conferencia "Enseñar para la Inteligencia". Nueva York. [en línea] Disponible en: http:// personales.com/mexico/tuxlagutierrez/ EDUNACH/LOS\%2OPARADIGMAS\%2 oVIGOSTKIANOS\%20Y\%20DE\%20EX PERIENCIA\%20DE\%20APRENDIZAJE \%20MEDIADO.htm [consulta 2006, 18 de febrero].

Anderson, J. R. (2001). Aprendizaje y memoria: Un enfoque integral ( $2^{\mathrm{a}} \mathrm{ed}$.). México: McGraw-Hill.

Benveniste, E. (1991). Problemas de lingüística general. Cap.V. Categorías del pensamiento y de la lengua. Cap. VI Comunicación animal y lenguaje humano. México: Siglo XXI Editores.

Berger, P.; Luckman, T. (1998). La construcción social de la realidad: El lenguaje y el conocimiento en la vida cotidiana. Bs. As. Argentina: Amorrortu Editores.

Bruner, J. (1991). Actos de significado, más allá de la revolución cognitiva. Cap. III. Madrid: Alianza Editorial.

Díaz-Barriga, F.; Hernández, G. (2002). Estrategias docentes para un aprendizaje significativo: Una interpretación constructivista ( $2^{\mathrm{a}}$ ed.). México: McGraw-Hill.

Driscoll, M.P.(2000). Psychology oflearning for instruction. Boston: Allyn \& Bacon.
Fromm, E. (1972). El lenguaje olvidado. Argentina: Librería Hachette.

Gardner, H. (1987). La nueva ciencia de la mente: Historia de la revolución cognitiva. Barcelona: Paidós.

Good, T. L.; Brophy, J. (1996). Psicología educativa contemporánea ( $5^{\mathrm{a}}$ ed.). México: McGraw-Hill.

Jochems, W.; Van Meriiënboer, J.; Koper, R. (2004). Integrated ELearning. Implications for pedagogy, technology \& organization. Londres: RoutledgeFalmer.

Johnson, D.; Johnson, R. (1999). Los nuevos círculos del aprendizaje. Bs. As. Argentina: Aique.

Knowles, M. S.; Holton, E. F.; Swanson, R. A. (2001). Andragogía: El aprendizaje de los adultos. México: Oxford University Press.

Jean, L.; Etienne, W. (2002). Situated Learning. Cambrige: UK.

Lewis, R. (2002). Grupos de trabajo en comunidades virtuales. Jornadas de la red FREREF NTIC. UOC: Catalunya. [en línea] Disponible en: http://www. uoc.edu/web/esp/art/uoc/lewiso102/ lewiso102.html [consulta 2006, 29 de enero].

Lockwood, F.; Gooley, A. (2001). Innovation in Open \& Distance Learning. Londres: Bogan Page Ltd.

Lockwood, F.; Gooley, A. (2001). Innovation in Open and distance learning. London: Kogan Page.

Mena, M. (2005). El diseño de proyectos de educación a distancia. Páginas en construcción. Bs. As.: La Crujía.

Mena, M. (2004). La educación a distancia en America Latina. Bs. As.: La Crujía.

Pazos, M.; Pérez, A.; Salinas, J. (2002). Comunidades virtuales: De las listas de discusión a las comunidades de aprendizaje. Universidad de las Islas 
Baleares. [en línea] Disponible en: http:// www.imacmexico.org/file download. php?location $=S$ U\&filename $=\mathbf{1 1 2 3 5 1 9 5}$ 271CVIRTUALES01.pdf [consulta 2006, 21 de febrero].

Preece, J. (2002). Online Communities: Designing Usability, Supporting Sociability. USA: John Wiley \& Sons. Ltd.

Preece, J. (2003). Online Communities. NY: Wiley.

Rowntree, D. (1992). Exploring Open and Distance learning. London: Kogan Page.

Saint-Onge, H.; Wallace, D. (2003). Leveraging Communities of Practice for Strategic Advance. Bruselas: Ed. Butterworth Heinemann.

Salmon, G. (2004). E-moderating: The Key to Teaching and Learning Online. (2 ed). London: Taylor \& Francis.

Salmon, G. (2003). E-tivities. London: Kogan Page.

Santrock, J. W. (2001). Psicología de la educación. México: McGraw-Hill.

Schunk, D. (1997). Teorías del aprendizaje ( $2^{\mathrm{a}}$ ed.). México: Prentice-Hall.
Segal, H. (1995). Sueño, fantasma y arte. Cap. III. Bs. As. Argentina: Ed. Nueva Visión.

Tarpy, R. (2000). Aprendizaje: Teoría e investigación contemporánea. Madrid: McGraw-Hill.

Teare, R.; Davies, D.; Sanderlands, E. (2002). Organizaciones que aprenden y formación virtual. Barcelona: Ed. Gedisa.

Tiwana, A. (2002). The Knowledge Management Toolkit. Londres: PrenticeHall.

Vigotsky, L. S. (1982). Pensamiento y lenguaje. La Habana. Cuba: Ed. Pueblo y Educación.

Wenger, E. (1998). Communities of Practice. Cambridge: University Press.

Wenger, E.; Lave, J.(1991). Situated learning . Cambridge: University Press.

Wenger, E.; Mc Dermott, R.; Snyder W. (2003). Cultivating Communities of practice. USA: Harvard Press.

Woolfolk, A. E. (1999). Psicología educativa ( $7^{\mathrm{a}}$ ed.). México: Pearson.

\section{PERFIL ACADÉMICO Y PROFESIONAL DE LAS AUTORAS}

Susana Trabaldo. Licenciada en Administración de Educación Superior (UNLM). Diploma de Postgrado en Educación a Distancia (CAECE), Máster en Enseñanza y Aprendizaje Abiertos y a Distancia (UNED, España). Directora de NetLearning, Entornos Virtuales de Aprendizaje. Capacitadora en diversos proyectos de e-learning. Docente universitaria. Directora de la Diplomatura en Diseño y Gestión de proyectos de e-learning (Universidad de San Martín), Directora de Experto Universitario en implementación de Proyectos de e-learning (UTN FRBA).

E-mail: strabaldo@net-learning.com.ar

Nancy Piriz. Profesora y Licenciada en Ciencias de la Educación, UBA. Especialista en Didáctica. Master en Enseñanza y Aprendizajes Abiertos y a Distancia (UNED, España). Posgraduada en Formación de Formadores UOC, Catalunya. Ex Directora del Colegio Cardenal Newman. Capacitadora de docentes y directivos. 
Docente universitaria. Directora de Net-Learning. Colaboradora de la Cátedra UNESCO de Educación a Distancia. Co-Directora de la Diplomatura en Diseño, Gestión y Evaluación de Proyectos de e-learning y Formación Virtual, Universidad Nacional de General San Martín.

\section{E-mail: npiriz@net-learning.com.ar}

Graciela Lorenzatti. Licenciada en Ciencias de la Educación con Especialidad en Planeamiento, Supervisión y Administración Educativa (Universidad Católica de Córdoba). Profesora de Lengua y Literatura Española (Universidad Nacional de Córdoba). Diploma de Postgrado en Gestión de proyectos de e-learning. Capacitadora de docentes y directivos en temas de EaD.

DIRECCIÓN DE LAS AUTORAS:

Av. Gaona 1810.

Código Postal : 1704. Ramos Mejía.

Buenos Aires. Argentina

www.net-learning.com.ar

Fecha de recepción del artículo: 01/03/07

Fecha de aceptación del artículo: 28/02/08 